\title{
Pediatric Atlas of Ultrasound- and Nerve Stimulation-Guided Regional Anesthesia
}

\author{
Ban C.H. Tsui, Santhanam Suresh (Eds). Springer, New York, 2016, 553 pages. \\ ISBN 978-0-387-79963-6
}

\author{
Arie Peliowski, MD
}

Received: 1 December 2015/Revised: 8 December 2015/Accepted: 15 December 2015/Published online: 4 January 2016

(C) Canadian Anesthesiologists' Society 2016

Pediatric regional anesthesia has experienced a renaissance in recent years. With the development of improved and more pediatric-specific needles and catheters, along with advancements in the quality and widespread adoption of ultrasound imaging by anesthesiologists, regional techniques are achieving higher success rates with better safety profiles. With this textbook, Tsui and Suresh aim to provide a comprehensive atlas that will shorten the learning curve for anesthesiologists seeking to improve their regional anesthesia skills.

Part I is a detailed guide of the equipment necessary for regional anesthesia - how it works and how to use it. Chapters describing the electrophysiology of nerve stimulation and the physics of ultrasonography are exemplary in that the authors have addressed complex topics and explained them in a manner that is understandable and easily digestible by clinicians.

Part II examines the specific clinical considerations unique to pediatric patients undergoing regional anesthetic techniques. The chapter describing the assessment of pain in children provides a good summary of the various scales and tools available as well as the full spectrum of other considerations that can affect the assessment of pain in children. The chapter on pharmacology relays the important differences that must be addressed when giving anesthesia to children. Interestingly, although it includes the relatively newer levobupivacaine and ropivacaine in tables describing the physical properties of various local anesthetics, the recommended doses of these newer agents

\footnotetext{
A. Peliowski, MD ( $₫)$

Department of Anesthesia and Pain Medicine, The Hospital for

Sick Children, Toronto, ON, Canada

e-mail: arie.peliowski@sickkids.ca
}

are absent from the table that includes recommended dosages. The chapter on complications is well organized and practical. Curiously, in a table of patient selection factors, it includes anatomic anomalies as a relative contraindication, but the authors do not elaborate any further. For example, the presence of a sacral dimple as a possible marker for occult spinal dysraphism - a possible contraindication to one of the most common regional techniques (i.e., caudal block) - is not mentioned anywhere in the book.

Part III of the textbook provides an excellent review of the anatomy relevant to regional anesthesia techniques. The illustrations are numerous and of excellent quality.

The remainder of the textbook (Parts IV-XI) focuses on what would likely most interest the readers: the regional anesthetic techniques themselves. Blocks for every part of the body are described in a well-organized, consistent manner. First, the relevant anatomy is described using both text and images. Next, landmarks, nerve stimulation, and ultrasound techniques are described in practical terms, including patient positioning, anatomic landmarks, how and where to insert needles, what to look for with nerve stimulation and ultrasound, and common errors and how to correct them. A typical, relevant clinical scenario is included for each category of block. Finally, each chapter concludes with a comprehensive reference list and suggested reading.

Last, but certainly not least, is the visual component of the atlas. It uses a combination of photographs, magnetic resonance images, visible human visualization software, and ultrasound images in a remarkably effective manner. All the images are of extremely high quality and clearly labelled. The editors have made the wise choice to use realworld ultrasound images that can be achieved by the 
typical user, rather than anatomically perfect images that may not be possible for every patient. There are typically two ultrasound images, one labelled and one not. This design is extremely helpful as it allows the reader to see the image uncluttered by labels. It also, however, provides a guide to the relevant structures, which are labelled, so readers can learn to identify the structures themselves.

In summary, this book is an excellent guide to regional anesthetic techniques in children. It gives a straightforward, easy-to-understand explanation of the important theoretical aspects as well as detailed, useful descriptions of the practical components of regional anesthesia. The images and visual components are outstanding. Although there are some curious but minor omissions, their absence does not detract from the overall quality of the book. This atlas is suitable for anesthesiologists at all levels of experience. Both trainees and consultants looking to learn about regional anesthesia will find this textbook a valuable resource. Even experienced pediatric regionalists could expand their repertoire with some of the more exotic techniques described.

Conflicts of interest None declared. 